\title{
PLUG-INS JUNGLE
}

\section{Algorithmic Design as Inbuilt Dynamism Between Human and Artificial Creativity}

\begin{abstract}
The paper seeks to define the concept of plug-ins jungle as generative background in computational design for new forms of dynamic relationship between human and artificial creativity. This definition will be conducted through the use of theoretical references and practical case studies with the intent to show that the current evolution of a plug-ins jungle is affecting design results at all its scales - from product design to urban design - and at all its stages - from design conception to construction and fabrication.
\end{abstract}

In the past twenty years, the creation of software applications during the design process has exponentially increased in every design-related disciple and the use of plug-ins is giving birth to a new algorithmic evolution of creativity. One of the most interesting examples regarding such matter corresponds to the graphical algorithmic editor Grasshopper and the generation of a series of animal-named plug-ins through which graphically editing the coding process. Grasshopper and its plug-ins represent the touchstone of the ongoing evolution of a new digital jungle in which new software applications are not only augmenting human creativity, but they are also generating new forms of artificial creativity in themselves.

The evidence suggests that the increasing use and creation of software applications during the design process is giving birth to new forms of dynamic relationship between human and artificial intelligence, an inbuilt dynamism through which the human mind and the machine constantly interact with each other producing unexpected and emergent design results.

\section{Keywords}

Plug-Ins, Algorithmic Design, Human Creativity, Artificial Creativity, Design Ethic

\section{Introduction}

In 2014, Andrew A. Smith publishes his book Grasshopper Jungle [1]. Despite being a young adult fiction and coming-of-age novel quite unusual and original for its literary genre, its title sounds particularly familiar if referred to algorithmic design and robotic fabrication. In fact, the graphical algorithmic editor Grasshopper currently represents one of the most popular and developed algorithmic editors for designers available on the market. Its use is widely spread throughout the majority of design-related disciplines which are now developing new algorithmic techniques to explore new ideas and opportunities during the design process. The rise of such algorithmic approach is transversally affecting the design process at all its scales, breaking down boundaries between disciplines which were far from each other during the past years. As a 
matter of fact, from urban design to product design, algorithms are providing new platforms of research contributing to the generation of emergent and autonomous design results.

The popularity of an algorithmic editor such as Grasshopper lies on a series of opportunities which it is able to provide to its users. First of all, Grasshopper allows the creation of graphical-based algorithms generated by an inductive process which allows the user to intuitively connect pre-coded nodes. Secondly, it allows the user to personally code new scripts through the use of several programming languages such as C\#, Python, and VB. Finally, the generation of new and totally customized plug-ins is then promoted inside a unique platform in which the same plug-ins can interact with each other. The inbuilt connection with a 3D modeling software such as Rhinoceros ultimately provides the user with the opportunity to physically produce the designed object through several production techniques, such as the creation of STL files for 3D printing or specific scripts to program robotic arms.

The innovation which this approach is bringing into the design process is clear and significant, and it is giving birth to new forms of relationship between human and artificial creativity. These new forms of evolution require an analysis able to consider algorithms not only as mere mathematical tools, but rather as examples of the rise of a new human progress in design conception based on the influence of artificial intelligence over human mind and perception.

Nowadays, the interaction between human and artificial intelligence represents an open field of debate and investigation, and such ongoing discussion about people in favor of or against to one side or the other has been well depicted by Max Tegmark in his book Life 3.0. Being Human in the Age of Artificial Intelligence [2]. In such a background of interest and research, a series of questions arise: what is the nature of this new relationship between human and artificial intelligence? How is it affecting the design process and the new forms of creativity generated from it?

The answer to these two questions requires the use of two concepts, one theoretical and one practical. The former is related to the theoretical point of view to adopt in evaluating the relationship between human and artificial creativity, while the latter refers to the practical ways through which such relationship is manifesting itself. The first concept is the concept of inbuilt dynamism between human and artificial intelligence, while the second one corresponds to the idea of plug-ins jungle. Since the former represents the theoretical point of view through which looking at the latter, a note on the inbuilt dynamism between human and artificial creativity is required before explaining the factors which are influencing the rise of the current plug-ins jungle.

\section{Note on the Inbuilt Dynamism Between Human and Artificial Creativity}

The expression 'inbuilt dynamism' refers to the analysis done by the cognitive scientist Margaret Boden in regards to the work of Harold Cohen and his Al system AARON, one of the first example of computer-generated art developed by the British artist during the 1970s. Commenting on Cohen's work and developing the same theoretical construction in her book The Creative Mind. Myths and Mechanisms [3], Boden highlights the fact that computational processes including scripts, frames and semantic nets - are helpful to understand how the brain works and how some aspects of human creativity are possible. The reason for it is "because symbolic and representational structures and transformations are the focus of computer programming, the essence of creativity may not be so far removed from computational processes as is usually assumed" [4]. Although the existance of similitudes between the human brain and computer was something already well presented in the studies of computational pioneers such as Alan Turing and John Von Neuman [5], Boden provides an interesting interpretative key through which reading such complementary relation between artificial and human intelligence:

A functioning program has its own inbuilt dynamism. Its activities can be both flexible and constrained, and a proper amalgam of flexibility and constraint is central to creative intelligence. [6]

Then, flexibility and constraint as the two main factors to understand the real measure of complementarity between artificial and human intelligence, a balance 
which can only be fully appreciated through the awareness of their inbuilt dynamism.

Another important contribution to the idea of dynamic complementarity between human and artificial creativity is provided by Kostas Terzidis. In his book Algorithmic Architecture Terzidis focuses the attention on the new computational process in architecture describing the use of algorithms not only as mere step-by-step problem-solving procedures, but rather as ontological constructions with philosophical and sociological repercussions. Starting from clarifying the distinction between computation and computerization - something already well explained by the author in his previous publication talking about 'algorithmic form' [7] Terzidis coined the term 'algotecture' to highlight the use of algorithms in architecture. Proceeding through the explanation of a brief history of such 'algotecture' as something substantially different from the more common Computer-Aided Design system - the former not necessarily dependent on the computer, while the latter dependent by definition on it - Terzidis gives evidence to the fact that there are certain levels of problems which cannot be solved by standard CAD systems, but their complexity inevitably required the use of algorithms. His words are particularly significant in this regard:

There are some problems whose complexity, level of uncertainty, ambiguity, or range of possible solutions required a synergic relationship between the human mind and a computer system. Such synergy is possible only through the use of algorithmic strategies that ensure $a$ complementary and dialectic relationship between the human mind and the machine. [8]

Such 'complementary and dialectic relationship between the human mind and the machine' highlighted by Terzidis explains the role of algorithmic design in comparison with human creativity through a critical point of view which can be reasonably reconducted to Boden's concept of inbuilt dynamism in functioning programs and their creative intelligence. This critical point of view is based on mutual persuasion and compromises which are able to generate new forms of creative dynamism inbuilt in the new artificial dimension of human creativity.
The analysis of these theoretical references has been conducted with the purpose to clarify the complementary relationship between human and artificial creativity. Such point of view represents the foundation upon which looking at algorithmic design and evaluating the results coming from it. The ongoing phenomenon of mathematization of the design process is based on the creation of software applications and this approach is characterizing the most advanced researches in contemporary design. New plug-ins are created and grouped together inside bigger platforms - such as Grasshopper - and sometimes they are even programmed to constitute stand-alone applications. Both cases represent the evidence of an exponentially increasing 'plug-ins background' which is progressively evolving the traditional conception of human design: this wide and open landscape is represented by the plug-in jungle.

\section{Plug-ins Jungle}

The expression plug-ins jungle refers to AD Profile 222 Computation Works. The Building of Algorithmic Thought guest-edited by Brady Peters and Xavier De Kestelier in 2013. Inside the issue, a series of examples are presented to analyze the effects of computational design inside the architectural discipline. In the article Design Eco-System. Customizing the Architectural Design Environment with Software Plug-ins, Daniel Davis and Brady Peters describe the evolution of architectural design towards the use of scripting and personalized plug-ins:

This defines an entirely new landscape in which 'cathedrals' (monolithic applications) are challenged by 'bazaars' (generative-modeling editors) populated by animal-named plug-ins. [9]

In the following pages, a series of Grasshopper plugins are described such as Kangaroo, Pachyderm Acoustic Simulation, Weaverbird, Geco, Firefly. If now we add to them other Grasshopper plug-ins such as Goat or Bowerbird, just to mention a couple - and even compound software such as Rhinoceros and its rendering extensions - Penguin or Flamingo among the others - the definition of a plug-ins jungle seems pertinent and appropriate for a computational environment dominated by animal-named applications. 
Inside the same AD Profile 222, a series of case studies are proposed with the purpose to highlight the effects of such algorithmic tools over the final design results. In this regard, particularly interested are the examples explained by Giulio Piacentino and the use of the Grasshopper plug-in WeaverBird [10], and Thomas Grabner and Ursula Frick and the use of another Grasshopper plug-in such as GECO [11]. In the first case, the use of the plug-in gives access to surface subdivisions and transformation operators which allow a topological editing of the starting design creation. In doing so, the exploration of a series of design options comes from the action performed by the algorithm itself, which therefore becomes a fundamental factor in design creation. In the second case, the use of the plug-in GECO in conjunction with the software Autodesk Ecotect allows a constant interaction between modeling and software analysis, and such interaction produces effects in terms of design results - such as in the case study analyzed in the article, that is the Shenzhen Boarder Station competition entry designed by SPAM, where the plug-in has been used to run a solar access analysis through which roof openings have been located and designed. In both cases, the use of plug-ins significantly influences the final design results, and therefore they are clear examples of how the action of the plug-ins jungle is a touchstone of the rising phenomenon of hybridization between human and artificial intelligence in the current evolution of creativity in computational design.

The examples which can be mentioned to confirm such fundamental influence of plug-ins over final design results are numerous and almost unlimited. In fact, nowadays the use of Grasshopper and its plugins is becoming part of the standard practice in many architectural firms, and it is very often the case to see such applications embedded into the workflow from the very early stages of the design process constituting basic tools of design exploration and investigation. Having said that, the concept of plug-ins jungle goes far beyond being a definition related to a single algorithmic editor such as Grasshopper, but rather it represents a more complex working model towards which both the design profession and the construction industry are evolving too. Algorithmic creations and software applications constitute by now the core of any advance research related to the built environment, and this aspect represents a clear confirmation of the fact that the ongoing process of mathematization is not only producing new forms of development inside the single discipline, but more importantly it is linking together different disciplines inside new algorithmic platforms.

\section{Extended Examples of Plug-Ins Jungle}

In the current evolution of computational design, there are several fields of research in which the action of the plug-ins jungle plays a central role in the realization of final design results.

First of all, plug-ins jungle is the natural habitat for the complexity of design generated in the last twenty years across all design-related disciplines. Promoted by the rise of a mass-customized production system, sophisticated software applications and new digital fabrication technologies have enables designers to build experimental structures with a high level of complexity. Such complexity is based on continuous and seamless forms, and although nowadays such trend seems to be overtaken by the rise of a more discrete approach - in this regards, Lei Zheng offers an interesting comparison between the two models [12] - the digital turn of design in the last two decades has been based on a constant exploration of complex shapes and structures. For instance, the work conducted at ZHACODE is particularly significant in this regard. The computation and design group at Zaha Hadid Architects is a research group which focuses its attention on invention and innovation through the use of computer software programming and physical computing, considering algorithms as exploratory phases for the discovery of new design and production opportunities. Among a wide range of projects, the installation 'Thallus' exhibited in Milan during Salone del Mobile 2017 represents a perfect example of the research conducted by ZHACODE in terms of customization and mechanization of the design process. The installation is composed by a $7 \mathrm{~km}$ long continuous line made of an extruded structural strip produced by 6 -axis robotic $3 D$ printing technology. The design of the structural strip is based on differential growth methods dictated by an algorithm which uses specific parameters - such as proximity to boundaries, angled direction of ruling, and structural requirements - to establish density gradation and direction of growth. 
Other than representing the field of action for complex forms and structures in digital mass-customized production, the plug-ins jungle is also the place for the creation of tools through which promoting opensource design. The rise of open-ended and adaptable systems based on sharing software, ideas and models is becoming one of the main field of research in the current evolution of computational design throughout all the disciplines. An interesting example of this approach is represented by the work done by Enriqueta Llabres and Eduardo Rico in terms of urban analysis and design. Through their multidisciplinary Londonbased office Relational Urbanism, Llabres and Rico developed a design approach to digital forms of urban documentation based on Relational Urban Models (RUMs). As explained by the authors in their article Relational Urban Models. Parameters, Values and Tacit Forms of Algorithms [13], RMUs are design models based on the new forms of digital urban documents which collect inputs from designers, government bodies and members of the public, allowing information sharing and feedback from the end user to the design team and vice versa. This new form of shared authorship in design conception is possible through an application based on a generative algorithm able to analyses data and parameters to elaborate new urban configurations. For instance, parameters based on the proximity of the street network allow to retained certain blocks and to remove certain others, or again the final configuration of a tower can be sculpted on top by constraints of sunlight exposure. The final design is the result of the action of algorithms able to mediate the effects of environmental parameters with the starting requirements coming from human inputs.

Another field of research in which the plug-ins jungle plays an important role is automation and digital production. For example, the wide use of 6 -axis robotic arms in design production and assembly is made possible by the use of algorithms and software applications which not only allow to program robots according to specific design requirements, but also influence the final design results through the control of the robot's action and movement. For instance, the Grasshopper plug-in Robots is a clear example of this process and it allows programming $A B B, K U K A$ and $U R$ robots. Always talking about robotic fabrication and the influence on design played by algorithms to program such machines, an interesting example is provided by the heterogeneous multi-robot systems which constitute an open field of research at the ICD/ITKE at the University in Stuttgart. As explained by Maria Yablonina and Achim Menges [14], such multi-robot systems are based on the use of bespoke design machines in conjunction with standard industrial robots. This mix during the fabrication process allows a higher level of flexibility and scalability compared to the exclusive use of industrial robotic arms. An example of this process is provided by the ICD/ITKE Research Pavilion 2016-17. In this case, the use of a path correction algorithm relying on camera-based tracking system controls the flight of the mobile robot which is represented by an unmanned aerial vehicle (UAV), while other software applications are used for analysis and optimization of the Pavilion structure dictating robots' movement through the location of the primary and secondary structures, both made of carbon tensile filament structures with the primary one realized in carbon fiber-reinforced ribs.

Finally, another field of research in which the action of the plug-ins jungle plays an important role in the creation of final design results is represented by optimization and form-finding techniques. As already explained by Mark Burry describing the works of Antoni Gaudí and Frei Otto as main precursors in computational design in terms of form-finding and structural optimization [15], nowadays such techniques are widely used in contemporary design conception and they are based on several software applications. For instance, Kangaroo is one of the most popular plug-ins for Grasshopper and its use allows to modify design in response to engineering analyses simulating aspects of the behaviour of real-world materials and objects. Always regarding such matter, the work of the Digital Structures research group at the MIT Massachusetts Institute of Technology represents an important example in terms of structural optimization and the different design configuration generated from it. Group leader Caitlin Mueller pays particular attention to the relationship between structural optimization and design conception in her article Distributed Structures: Digital Tools for Collective Design [16]. Highlighting the fact that the creation of new computational tools is shifting the role of computation itself from representation and analysis to creative idea generation, Mueller provides a series of examples in which the use of multi-objective 
optimization techniques offers the opportunity for the designer to choose between different options generating during the optimization process. Such opportunities are made possible to the use of plug-ins specifically developed for such purpose. For instance, the web-based design application StructureFIT and the Rhino and Grasshopper plug-in Stormcloud - both developed by the MIT Digital Structures research group - allow to designers to explore new design typologies and forms with high level of structural feasibility.

The examples described so far represent only four instances belonging to a wide range of case studies in which the final design is the result of a very close relationship between the human input and the computational calculation belonging to the machine. From this relationship, a new design world is rising and the plug-ins jungle represents its natural habitat. Such habitat is based on the constant interaction between the human mind and the machine which effects design right at the very beginning of its conception. Inside such contextual background, plug-ins are the places able to host new forms of emergent creativity coming from a new relationship between the man and the machine.

\section{Conclusion}

The theoretical considerations and practical case studies analyzed so far represent a critical point of view through which looking at the current evolution of computational design and the increasing use of software applications during the design process. The rise of the plug-ins jungle is affecting computational design at all its scales - from product to urban design - and at all its stages - from design conception to automated fabrication. Software applications are becoming indispensable prerequisite for any design process and the fact that the authorial role of the human designer may not survive to this digital turn is a concrete possibility for the future of any designrelated profession. As already explained by Mario Carpo in his book The Alphabet and the Algorithm, the more frequenwt use of algorithms may eventually transform human designers into IT developers, i.e., changing their role from creators to mere plug-ins users/generators [17]. This scenario may not represent an alarming point for those ones who believe that the future will not belong to human beings but to machines. On the other hand, this point of view may not be supported from those ones who believe that any technological development is first and foremost a matter of human progress.

The theoretical construction of the entire paper is built upon the association between two apparently dissimilar species - such as the man and the machine - and the inbuilt dynamism which regulates such relationship. Considering the design process as a field of investigation has constituted an appropriate point of view to understand the dynamic complementarity between such species and the creativity which arises from them. After all, as already explained by Nicholas Negroponte at the very beginning of the computational evolution of design:

The partnership is not one of master (smart, leader) and slave (dumb, follower), but rather of two associates which each have the potential for self-improvement [...]. Eventually, a separation of the parts could not occur; the entire 'symbiotic' system would be, as Gordon Pask described, an artificial intelligence that cannot be partitioned. [18]

According to Negroponte, from the rise of this new form of artificial intelligence, a new 'extended designer' (the man) and 'artificial designer' (the machine) will be generated in favor of a constant and mutual design complementation, augmentation, and interaction between the human mind and the machine. Today, such 'extended designer' and 'artificial designer' are two actors of the same reality and their interaction is constantly producing new forms of inbuilt dynamism between human and artificial intelligence. The complementary approach between them seems to be a more appropriate point of view from which looking at the ongoing evolution of algorithmic design, and a closer look into its natural habitat, namely a walk in the plug-ins jungle, may provide an effective starting point for further research.

\section{References}

1. Smith, A. (2014). Grasshopper Jungle. Penguin Books.

2. Tegmark, M. (2017), Life 3.0. Being Human in the Age of Artificial Intelligence. Penguin Books, p.31.

3. Boden, M. (1990). The Creative Mind. Myths and Mechanisms. Weidenfeld and Nicolson. 
4. Boden, M. (1983). Creativity and computers. In Harold Cohen. Exhibition catalogue. The Tate Gallery, p.17.

5. Von Neuman, J. (1958). The Computer and the Brain. Yale University Press.

6. Boden, M. (1983). Creativity and computers. In Harold Cohen. Exhibition catalogue. The Tate Gallery, p.18.

7. Terzidis, K. (2003). Algorithmic Form. In Expressive Form. Spon Press, pp.65-75.

8. Terzidis, K. (2006). Algorithmic Architecture. Architectural Press, p.37.

9. Davis, D. \& Peters, B. (2013). Design Eco-System. Customizing the Architectural Design Environment with Software Plug-Ins. In Computational Works. The Building of Algorithmic Thought, guest-edited by Peters, B. \& De Kestelier, X. AD Profile 222 (March-April 2013), p.125.

10. Piacentino, G. (2013). WeaverBird. Topological mesh editing for architects. In Computational Works. The Building of Algorithmic Thought, guest-edited by Peters, B. \& De Kestelier, X. AD Profile 222 (March-April 2013), pp.140-1.

11. Grabner, T. \& Frick, U. (2013). GECO. Architectural Design Through Environmental Feedback. In Computational Works. The Building of Algorithmic Thought, guest-edited by Peters, B. \& De Kestelier, X. AD Profile 222 (March-April 2013), pp.142-3.

12. Zheng, L. (2019). Meta-Utopia and the Box. Two Stories about Avant-Garde Projects. In Discrete. Reappraising the Digital in Architecture, guest-edited by Retsin, G. AD Profile 258 (March-April 2019), pp.130-5.

13. Llabres, E. \& Rico, E. (2016). Relational Urban Models. Parameters, Values and Tacit Forms of Algorithms. In Parametricism 2.0. Rethinking Architecture's Agenda for the 21st Century, guest-edited by Schumacher, P. AD Profile 240 (March-April 2016), pp.84-91.

14. Yablonina, M. \& Menges, A. (2019). Distributed Fabrication. Cooperative Making with Larger Groups of Smaller Machines. In Discrete. Reappraising the Digital in Architecture, guest-edited by Retsin, G. AD Profile 258 (March-April 2019), pp.62-9.

15. Burry, M. (2016). Antoni Gaudí and Frei Otto. Essential Precursors to the Parametricism Manifesto. In Parametricism 2.0. Rethinking Architecture's Agenda for the 21st Century, guest-edited by Schumacher, P. AD Profile 240 (March-April 2016), pp.30-5.

16. Mueller, C. (2017). Distributed Structures. Digital Tools for Collective Design. In Autonomous Assembly. Designing for a New Era of Collective Construction, guest-edited by Tibbits, S. AD Profile 248 (July-August 2017), pp.94-103.
17. Carpo, M. (2011). The Alphabet and the Algorithm. MIT Press, pp.126-7.

18. Negroponte, N. (1969) Towards a Humanism through Machines. In Architectural Design n. 7/6 (September 1969), pp. 511-2 